\title{
Familiarity affects environmental sound processing outside the focus of attention: An event-related potential study
}

\author{
Ursula Kirmse *, Thomas Jacobsen, Erich Schröger \\ University of Leipzig, Institute for Psychology I, Seeburgstr. 14-20, 04103 Leipzig, Germany
}

\section{A B S T R A C T}

Objective: This study investigated the influence of the familiarity of an environmental sound on sound processing outside the focus of attention.

Methods: By comparing ERPs elicited by a familiar, animal sound and an acoustically matched, but unfamiliar, complex sound, three issues were addressed: (a) general differences in the processing of the familiar and the unfamiliar sound, (b) influences of sound familiarity on the processing of deviants unrelated

Keywords:

Familiarity

Environmental sounds

Auditory N1

P2

Sound context

Passive

to familiarity and (c) familiarity-specific processing depending on the sound context. Participants watched a silent, subtitled movie.

Results: The familiar sound elicited a centro-parietal enhancement of the N1, a frontocentrally enhanced P2 and an additional P250. Auditory deviance processing elicited by deviants in sound location was not influenced by the familiarity of the sounds. However, after an involuntary switch of attention to the deviant, an N400-like deflection indicated enhanced semantic analysis of the familiar deviant. Familiarityspecific ERP effects as a consequence of the sound context occurred between 300 and $500 \mathrm{~ms}$ after stimulus onset.

Conclusion: Whereas familiarity of an environmental sound elicited enhanced stimulus processing before $300 \mathrm{~ms}$, influences of the sound context were observed subsequent to $300 \mathrm{~ms}$.

Significance: Familiarity of a complex environmental sound influences several stages of auditory processing outside the focus of attention.

(c) 2009 International Federation of Clinical Neurophysiology. Published by Elsevier Ireland Ltd. All rights reserved.

\section{Introduction}

Environmental sounds, i.e. complex sounds invariably associated with a specific meaning, provide information about events and objects in the personal environment (cf. Ballas and Howard, 1987). Familiar environmental sounds are represented in auditory long-term memory, have semantic significance and are consciously identifiable. Their significance can be extracted without the need for visual exploration and is based on acoustic familiarity with the characteristic sound patterns typically elicited by specific events in the environment.

To date, the processing of environmental sounds has mainly been studied when sounds were processed attentively (e.g., van Petten and Rheinfelder, 1995; Lewis et al., 2005; Cummings et al., 2006; Lenz et al., 2007; Murray et al., 2008). However, as familiar environmental sounds might convey behaviorally and biologically relevant information, processing advantages for familiar

* Corresponding author. Tel.: +49 341 9735978; fax: +49 3419735969. E-mail address: ukirmse@uni-leipzig.de (U. Kirmse). sounds might be expected even when sounds are processed outside the focus of attention.

The present study used event-related potentials (ERPs) to address differences in the processing of a familiar environmental sound and an acoustically matched, but unfamiliar complex sound. Participants watched a silent movie as primary task and thus did not attend to the sounds. Previous studies indicate three aspects of sound processing outside the focus of attention to be susceptible to effects of sound familiarity: (1) sound processing in the time range of the auditory N1 and P2 component, (2) auditory deviance processing and (3) processing of sounds in their ambient sound context.

Firstly, previous studies indicated effects of sound familiarity already on relatively low-level stages of auditory processing. When presenting musical instrument tones or speech sounds, i.e. auditory stimuli that are less complex than environmental sounds, enhanced processing of familiar stimuli was observed in the $\mathrm{N} 1 /$ P2 time window. This time window is associated with the establishment of an auditory percept and its sensory memory representation (e.g., Näätänen and Winkler, 1999). Musicians, who are highly familiar with instrument sounds, were found to show enhanced $\mathrm{N} 1 \mathrm{~m}$ and $\mathrm{P} 2$ responses to instrument tones, and 
specifically to tones of their own instrument of practice, compared to non-musician controls (e.g., Pantev et al., 1998, 2001; Shahin et al., 2003, 2004) ${ }^{1}$. More recently, Ylinen and Huotilainen (2007) reported an enhanced N1 in the ERP elicited by vowels from the native language in contrast to language-unfamiliar vowels. Notably, an increase in the $\mathrm{N} 1(\mathrm{~m})$ and/or P2 response for non-intentionally processed sounds has also been observed intraindividually after acoustic discrimination training (e.g., Menning et al., 2000; Atienza et al., 2002; Tremblay and Kraus, 2002; Bosnyak et al., 2004). A similar increase in the P2 response observed after passive stimulus exposure (Sheehan et al., 2005) indicated that the observed effects were related to increased acoustic familiarity with the presented sounds.

Whereas the reviewed effects were observed when sounds were ignored, familiarity effects for complex environmental sounds were so far only addressed for attended sounds (e.g., Lenz et al., 2007; van Petten and Rheinfelder, 1995) or attention-capturing, "novel" sounds (e.g., Escera et al., 2003; Mecklinger et al., 1997). For the first time, the present study compared the processing of a familiar and an unfamiliar environmental sound when sounds were presented as regular, non-deviant items outside the focus of attention.

Secondly, several studies reported sound familiarity effects on automatic auditory deviance processing as reflected in the Mismatch negativity (MMN; e.g., Näätänen et al., 2007; Schröger, 2007; Winkler, 2007) and the P3a (e.g., Escera et al., 1998). The MMN is a negative deflection that occurs between 100 and $250 \mathrm{~ms}$ and is elicited by a violation of an auditory regularity even if participants ignore the presented sounds. If the violation is salient, the MMN is followed by a P3a. Both ERP components are best observed in the difference wave between the ERPs elicited by the sounds violating the auditory sequence, labeled deviants, and the sounds establishing the regularity, labeled standards. Whereas the MMN indicates the detection of the deviance as a result of a sensory memory comparison process between standard and deviant sounds (cf. Näätänen, 1992), the P3a is commonly taken to reflect an involuntary attention switch to the deviant [cf. e.g., Escera et al., 1998; however, see Horvath et al. (2008), who proposed a broader role of the P3a as an higher-level event-detection mechanism]. Regarding sound familiarity, several studies reported enhanced auditory deviance processing for familiar compared to unfamiliar deviant sounds [e.g., for native vs. non-native speech sounds (e.g., Näätänen et al., 1997); personally familiar vs. unfamiliar voices (e.g., Beauchemin et al., 2006); lexical items vs. pseudowords (e.g., Pulvermüller et al., 2001) or meaningful vs. non-meaningful environmental sounds (e.g., Frangos et al., 2005; Jacobsen et al., 2005)]. Additionally, Jacobsen et al. $(2004,2005)$ reported enhanced auditory deviance detection when deviants are presented in a sequence of familiar standard sounds, i.e. in a familiar context. Other studies observed effects related to the familiarity of a deviant or novel sound only subsequently to the MMN, either on the P3a (e.g., Escera et al., 2003; Neuloh and Curio, 2004; Holeckova et al., 2006; Wetzel and Schröger, 2007; for sound familiarity effects on novelty processing see also Cycowicz and Friedman, 1998) or outside the MMN/P3a complex (e.g., Mecklinger et al., 1997; Roye et al., 2007; Wetzel and Schröger, 2007).

Notably, all these studies observed familiarity-related effects on auditory deviance processing when different auditory items were presented as standard and deviant sounds, i.e. when the deviance corresponded to a change of the auditory item itself (e.g., Beauchemin et al., 2006; Frangos et al., 2005). However, if this is the case, the familiarity of standard and deviant sounds might directly influ-

${ }^{1}$ Differences in the results between magnetic field and ERP responses, showing effects on the N1m in the MEG and effects on the P2 in the EEG, seem to be due to the differential sensitivity of both methods to the orientation of generator structures (however, cf. Kuriki et al., 2006). ence the standard-deviant sensory-memory comparison process. The present study tested, whether sound familiarity likewise affects auditory deviance processing, when the presented deviance is unrelated to the familiarity of the sounds. To this end, deviants in sound location were presented separately for the familiar and the unfamiliar sound.

Thirdly, differences in the processing of a familiar and an unfamiliar environmental sound might occur depending on the familiarity of the auditory context in which the familiar and the unfamiliar sound are encountered. Existing data indicate that certain properties of the auditory context can be extracted even when participants do not attend to the auditory input (e.g., Sussman and Winkler, 2001; Winkler et al., 2003). Conversely, properties of the auditory context have been shown to influence task-related processing of individual sounds (e.g., Trainor and Trehub, 1993; see also Ballas and Mullins, 1991). Moreover, data of Jacobsen et al. (2004) indicate that a familiar sound context might influence the passive processing of single sounds. To address possible contextrelated effects, the present study contrasted the processing of the familiar and the unfamiliar sound when sounds were either presented in separate blocks (i.e. occurred in an either consistently familiar or consistently unfamiliar auditory context) or when both sounds were presented intermixed.

To summarize, the present study compared the processing of a familiar environmental sound and an acoustically matched, but unfamiliar complex sound outside the focus of attention in three conditions. Generally, we expect first effects of sound familiarity in the time range of the auditory N1 and P2 (cf. e.g., Pantev et al., 1998; Shahin et al., 2003). More specifically, overall effects of sound familiarity should be reflected in processing differences constant over the conditions. The comparison of location-deviant processing for the familiar and the unfamiliar sound should reveal possible effects of sound familiarity on the processing of a deviance unrelated to sound familiarity (i.e. sound location). Processing differences between separate and intermixed sound presentation should be reflected in context-dependent ERP effects.

\section{Methods}

\subsection{Participants}

Data were collected from 24 healthy young adults aged between 18 and 33 years (mean age $=22 ; 7$ years; 7 males). One additional participant was excluded from the data analysis due to a very high EEG artifact rejection rate (all conditions > 30\%). All participants gave their informed consent before the experiment and received monetary compensation or course credits for their participation. The experimental protocol conformed to the Declaration of Helsinki and the ethics guidelines of the German Association of Psychology (Ethics Board of the Deutsche Gesellschaft für Psychologie).

\subsection{Design}

Non-intentional processing of a familiar, meaningful environmental sound and an acoustically matched, but meaningless and unfamiliar complex sound was compared in three experimental conditions, which were presented to the participants outside the focus of attention.

(1) In the $100 \%$-consistent condition, the familiar stimulus and the unfamiliar stimulus were presented in separate blocks. Hence, sounds here were presented in a consistently familiar or consistently unfamiliar sound context, respectively. For each stimulus, 120 trials were presented. 
(2) In the Location-oddball condition, the familiar and the unfamiliar stimulus were presented in separate blocks as well. However, in this condition, randomly, $10 \%$ of the trials were presented from a different sound location (cf. Fig. 1). Note that the only feature in which deviants differed from standards was sound location and that hence the presented deviance was entirely unrelated to the familiarity of the sounds. As the familiar and the unfamiliar stimulus were presented in different blocks, not only the familiarity of the deviants, but also the familiarity of the standard sounds differed for location deviants presented for the familiar and the unfamiliar stimulus. For each stimulus, a total number of 1080 standard and 120 deviant trials was presented. Apart from differences in the amount of trials and the occurrence of deviants in sound location, the Location-oddball condition was very similar to the $100 \%$-consistent condition. For standard sounds, ERPs thus should largely replicate familiarity effects observed in the $100 \%$-consistent condition. On the other hand, deviant-minus-standard difference waves, calculated separately for the familiar and the unfamiliar sound, should reveal possible familiarity-related effects on the processing of a deviance unrelated to sound familiarity.

(3) In the 50\%-mixed condition, the familiar and the unfamiliar stimulus were presented randomly intermixed with 50\% probability each. For each stimulus, 120 trials were presented. In contrast to the $100 \%$-consistent and the Location-oddball condition, the 50\%-mixed condition did not feature an either consistently familiar or consistently unfamiliar context. Processing of both stimuli here can be compared when both stimuli occur in a common sound context.

\subsection{Experimental procedure}

Sounds were presented via loudspeakers (EventElectronis, TR6) placed in a distance of $1.30 \mathrm{~m}$ from the participant. One loudspeaker was positioned $30^{\circ}$ to the left, the other $30^{\circ}$ to the right hemifield (see Fig. 1). Participants attended to a silent, subtitled movie. The movie was presented on a computer monitor that was located between the two loudspeakers. Participants were instructed to ignore the presented sounds and to keep their gaze directed to the monitor.

Sounds were presented from one of the two loudspeakers respectively. Two blocks were presented per condition. In the $100 \%$-consistent and the 50\%-mixed condition, all sounds within one block were presented from one loudspeaker. Respectively

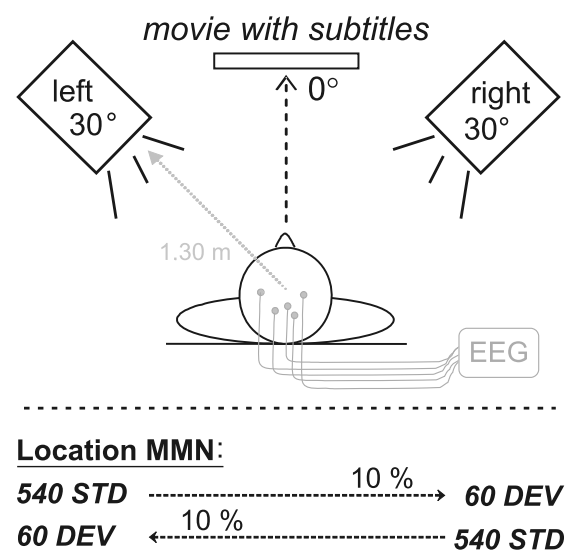

Fig. 1. Experimental setup. Participants were instructed to focus their attention on the silent video presented on the screen and to ignore the sounds presented via loudspeakers. one block of each condition was presented from the left loudspeaker, the other from the right one. In the Location-oddball condition, standards were presented from one loudspeaker, location deviants from the opposite loudspeaker. In one block, standards were presented from the left and deviants from the right hemifield; in the other block the assignment was opposite (cf. Fig. 1). Potential processing differences due to a lateralized sound or deviance presentation should thus average out in the average for each condition. Stimulus delivery was accomplished with the Cogent 2000 toolbox (running under Matlab 7.1; MathWorks GmbH, 2005). Sounds were presented with an intensity of $75 \mathrm{~dB}(\mathrm{~A})$ $[\mathrm{dB}(\mathrm{A})=$ a commonly used filter to adjust the Sound Pressure Level to the frequency characteristics of human hearing]. Trials were separated by a fixed inter-stimulus interval of $600 \mathrm{~ms}$ (sound-offset to sound-onset interval). Blocks were presented in a randomized order and breaks were offered according to the participants' individual needs. The total duration of the experiment was approximately $2 \mathrm{~h}$ including electrode application.

\subsection{Stimuli}

A familiar and meaningful environmental sound and an acoustically matched, but unfamiliar and non-meaningful complex sound were presented in the experiment (see Fig. 2). The familiar environmental sound was an isolated animal vocalization, the sound of a young sheep ("Bah-ah"). This stimulus (22.05 kHz sampling rate) had a length of $637 \mathrm{~ms}$ and was clipped from a longer sound file obtained from a freely-accessible, online, animal-sound database [www.seaworld.org/animal-info/sound-library/index.htm]. The unfamiliar stimulus was synthesized from the familiar stimulus using a multi-stage stimulus manipulation algorithm [data processing via Matlab 7.1 (MathWorks GmbH, 2005); for similar algorithms of stimulus manipulation cf. Lenz et al., 2007; Altmann et al., 2007]. After extracting the frequency spectrum of the familiar sound via a Fast-Fourier-Transformation (FFT), the obtained phase information, characterizing the temporal relationships between the frequencies contained in the sound, was randomly jumbled between the frequencies. However, amplitude values were

\section{(a) familiar stimulus unfamiliar stimulus}

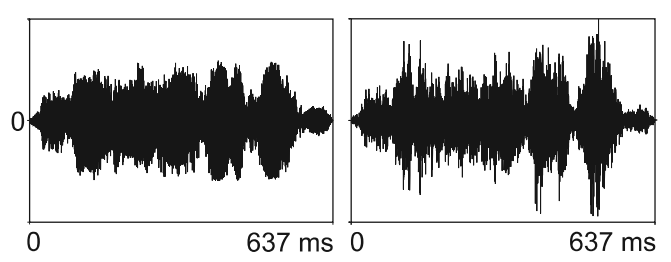

(b)

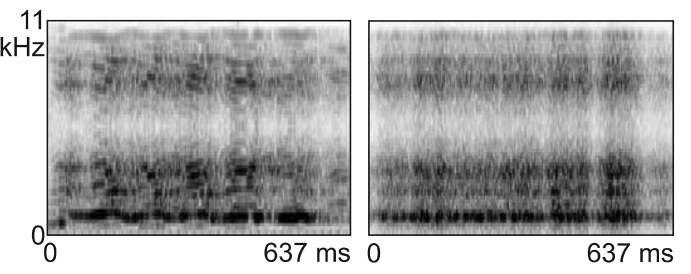

(c)

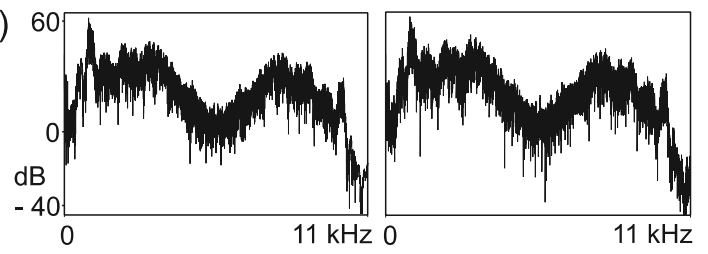

Fig. 2. Oscillogram (a), spectrogram (b) and frequency spectrum (c) of the familiar and the unfamiliar stimulus. 
kept for each frequency, so that each frequency was contained in the synthesized sound with the same intensity as in the original sound. Subsequently, a Hilbert transformation was used to extract the temporal envelope from the original sheep sound. The synthesized sound was then fit to this envelope. Finally, the intensity of both stimuli was normalized by adjusting the root mean square (RMS). Moreover, both sounds were additionally checked for comparable energy characteristics at the sound onset, as the sound onset is crucial for the auditory ERP. Altogether, the synthesized stimulus thus preserved the spectrotemporal characteristics of the original sheep vocalization (cf. Fig. 2), but could no longer be identified as a meaningful item. All parameters that in particular influence ERP components prior to $300 \mathrm{~ms}$ were kept equal between the two stimuli. Physically, the largest remaining difference was the harmonics-to-noise ratio (HNR), which is a measure of the harmonic content present in an auditory signal ${ }^{2}$.

The difference in perceived familiarity between the two stimuli was confirmed in a stimulus pre-test $(n=8)$ with participants that did not participate in the later EEG experiment. In this pre-test, both stimuli were evaluated together with a large range of further environmental sounds, containing 16 household sounds, 15 animal sounds and 15 complex, but unfamiliar sounds (synthesized with the same algorithm as described above). The two stimuli used in the present experiment had the highest dissociation in familiarity and identification measures of all tested familiar-synthesized sound pairs. The familiar stimulus was correctly named by all subjects with a high confidence (mean: $6.88 ; n=8 ; 1=$ not confident at all; 7 = very confident) and rated as very familiar (mean: 6.88; $1=$ not familiar at all; $7=$ very familiar). The unfamiliar stimulus was perceived as a non-meaningful burst of noise. No associations to a sheep and no repeated sound labels were given in the identification task for the unfamiliar stimulus and naming confidence for the given labels (e.g., "rattling sound") was very low $(2.25 ; n=4)$. The unfamiliar stimulus was rated with a familiarity of 2.25 .

\subsection{EEG recording and analysis}

Electroencephalographic (EEG) data were recorded with 32 active $\mathrm{Ag} / \mathrm{AgCl}$ electrodes and the BioSemiActive-Two acquisition system. Electrodes were positioned according to the extended International Ten-Twenty system and were mounted in a prefabricated cap. Data were sampled at $512 \mathrm{~Hz}$ (DC-coupled, $103 \mathrm{~Hz}$ physical lowpass filter). Two electrodes inherent to the BioSemi acquisition system [Common Mode Sense (CMS) and Driven Right Leg (DRL)] served for reference and ground purposes during the recording and were respectively located left and right posterior to $\mathrm{Cz}$. Further additional electrodes were placed at the tip of the nose and the left and right mastoid. The horizontal and vertical electrooculogram (HEOG/VEOG) were measured from the outer canthi of both eyes (HEOG) and supra- and infraorbital to the right eye (VEOG).

EEG data analysis was performed with EEP 3.3 (ANT Software B.V.). With EEP 3.3, data were first converted from BioSemi format (.bdf) to the compressed EEP file format (.cnt). Data were then offline re-referenced to the tip of the nose and were filtered with a $1-15 \mathrm{~Hz}$ bandpass filter. Trials including voltage signals exceeding $+/-75 \mu \mathrm{V}$ were excluded from the analysis $(17.5 \%$ of trials on average). ERPs with an epoch length of $700 \mathrm{~ms}$, including a $100 \mathrm{~ms}$ pre-stimulus baseline, were calculated separately for

\footnotetext{
${ }^{2}$ Animal sounds are typically characterized by a high HNR in contrast to other types of environmental sounds, as e.g., household sounds (cf. Lewis et al., 2005). As a consequence of the applied stimulus manipulation, the HNR was lower for the unfamiliar than the familiar stimulus [1.22 vs. $6.05 \mathrm{~dB}$ (determined using default parameters with Praat, freely available phonetics software, http://www.fon.hum.uva.nl/praat/)].
}

each participant, stimulus and the three conditions. For a better comparison of stimulus familiarity effects between the conditions, familiar-unfamiliar difference waves were calculated for each condition by subtracting the ERP elicited by the unfamiliar sound from the ERP elicited by the familiar sound (familiar-unfamiliar difference wave). For statistical purposes, mean amplitudes of the observed effects were quantified in each condition as the mean amplitude of the ERP elicited by the familiar and the unfamiliar stimulus over a grid of $3 \times 3$ electrodes $(F 3, F z, F 4, C 3, C z$, $C 4, P 3, P z$, and $P 4)$. These electrode locations adequately covered the maximal distributions of all relevant ERP effects and allowed assessment of each ERP effect's scalp distribution in a $3 \times 3$ factorial design (see below).

\subsection{Statistical data analysis}

ERP effects due to sound familiarity were observed and quantified in five different time windows: $100 \mathrm{~ms} \pm 15$ (N1), $165 \mathrm{~ms} \pm 15$ (P2), $231 \mathrm{~ms} \pm 15$ (P250), $343 \mathrm{~ms} \pm 15$ (Neg.300) and $417 \mathrm{~ms} \pm 15$ (Diff.400). For the N1 and P2, the respective time window was determined according to the respective component's mean peak maximum in the group grand-average. For later effects, time-windows were determined according to the maximum of the effect in the familiar-unfamiliar difference wave. Four-way repeatedmeasurement ANOVAs were calculated for each time window including the within-subject factors Condition (100\%-consistent; Location-oddball standards; 50\%-mixed); Stimulus familiarity (familiar; unfamiliar); Laterality (left; middle; right) and Anteriority (frontal; central; posterior). As only effects including the factor Stimulus familiarity indicate familiarity-related effects, only effects including this factor will be reported. While effects of Stimulus familiarity will reflect effects of the familiarity of the stimulus in general, interactions of Stimulus familiarity $\times$ Condition will indicate processing differences as an effect of the sound context, in which the stimuli occurred. Processing differences for a consistent versus intermixed sound context will be reflected in subsequent reduced ANOVAs if (a) stimulus processing and/or familiarity effects in the 50\%-mixed condition differ from processing in an consistently familiar or unfamiliar context (i.e., the $100 \%$-consistent condition as well as Location-oddball standards), while (b) processing does not differ between the $100 \%$-consistent condition and the Location-oddball standards. Deviants from the Location oddball condition were not included into these analyses, as deviance-related processing here superimposes familiarityrelated effects.

Location deviance processing (MMN/P3a) for the familiar and the unfamiliar stimulus was analyzed by quantifying the mean amplitudes of the ERP elicited by familiar and unfamiliar standard and deviant sounds at $130 \mathrm{~ms} \pm 15$ (mean MMN peak maximum) and $233 \mathrm{~ms} \pm 15$ (mean P3a peak maximum). Additionally, a sustained negativity was observed in the difference wave for the familiar but not the unfamiliar stimulus - and thus for the familiar, but not the unfamiliar deviant - and was quantified between 300 and $400 \mathrm{~ms}$. Mean amplitudes in these three time windows were compared in separate four-way repeated-measurement ANOVAs [within-subject factors: Oddball stimulus (deviant; standard) $\times$ Familiarity (familiar; unfamiliar) $\times$ Laterality (left; middle; right) $\times$ Anteriority (frontal; central; posterior)]. Only significant effects including the factor Oddball stimulus will be reported, as only these effects indicate modulations of the standard-deviant contrast. Sound familiarity effects on auditory deviance processing will be reflected in an Oddball stimulus $\times$ Familiarity interaction.

The significance level was set to $p \leqslant .05$ and Greenhouse-Geisser corrected degrees of freedom were applied and will be reported where the assumption of sphericity was not met. Relevant significant interactions were specified by calculating reduced ANOVAs 
separately for each level of the interacting factors. Bonferroni corrected $t$-test were used as post-hoc tests.

\section{Results}

Results will be reported and discussed in relation to the three addressed research questions and were observed in three partly overlapping time-windows: (1) overall differences in the processing of the familiar and the unfamiliar environmental sound were observed before $300 \mathrm{~ms}$ after stimulus onset, (2) auditory deviance processing of location deviants was observed between 100 and $400 \mathrm{~ms}$ and (3) ERP modulations as an effect of sound context familiarity were observed starting from $300 \mathrm{~ms}$.

\subsection{Effects related to sound familiarity}

ERPs elicited by the familiar and the unfamiliar stimulus respectively in the three experimental conditions are displayed in Fig. 3. Effects of Stimulus familiarity that were independent from the condition in which the sounds were presented were observed in three time windows.

The earliest effect of sound familiarity was observed in the time window of the N1. A main effect of Stimulus familiarity $\left(F_{1.23}=12.89 ; p<.01\right)$ here reflected an enhanced $\mathrm{N} 1$ amplitude for the familiar stimulus (see Fig. 3, cf. Table 1). Furthermore, a Stimulus familiarity $\mathrm{x}$ Anteriority interaction $\left(F_{2,46}=7.67\right.$; $p<.01)$ reflected a centroparietal scalp distribution of this effect (see Fig. 4). Reduced ANOVAs revealed a significantly enhanced $\mathrm{N} 1$ for the familiar stimulus at central and posterior electrode positions (central: $F_{1,23}=15.83 ; p=.001$; posterior: $F_{1,23}=14.99$; $p=.001$ ), but only a marginally significant effect at frontal electrode positions $\left(F_{1,23}=3.35 ; p=.08\right)$.

Similarly, the familiar stimulus elicited an enhanced P2 as reflected in a Stimulus familiarity $\times$ Anteriority interaction $\left(F_{2,46}=9.07 ; p<.01\right)$. In contrast to the effect observed for the $\mathrm{N} 1$, the P2 effect was constrained to frontocentral areas (see Fig. 4). Significantly larger P2 amplitudes for the familiar stimulus
Table 1

Mean ERP amplitudes (standard deviations) elicited by the familiar and the unfamiliar stimulus in the three conditions in the analyzed time windows.

\begin{tabular}{llccr}
\hline & & $\begin{array}{l}100 \% \text {-consistent } \\
\text { condition }\end{array}$ & $\begin{array}{l}\text { Location-oddball } \\
\text { standards }\end{array}$ & \multicolumn{1}{c}{$\begin{array}{l}\text { 50\%-mixed } \\
\text { condition }\end{array}$} \\
\hline N1 & familiar & $-0.31(1.45)$ & $0.32(1.04)$ & $-0.49(1.56)$ \\
& unfamiliar & $-0.11(1.66)$ & $0.73(0.90)$ & $0.45(1.20)$ \\
P2 & familiar & $2.20(1.49)$ & $2.71(1.14)$ & $1.96(1.62)$ \\
& unfamiliar & $1.84(1.68)$ & $2.15(1.06)$ & $1.92(1.25)$ \\
P250 & familiar & $1.38(0.94)$ & $1.35(0.73)$ & $1.83(1.85)$ \\
& unfamiliar & $0.72(1.43)$ & $0.43(0.71)$ & $0.84(1.21)$ \\
Neg. 300 & familiar & $-0.78(1.44)$ & $-0.30(0.53)$ & $0.07(1.06)$ \\
& unfamiliar & $0.29(1.27)$ & $0.12(0.56)$ & $-0.09(1.11)$ \\
Diff. 400 & familiar & $-0.06(1.23)$ & $0.18(0.44)$ & $0.90(0.95)$ \\
& unfamiliar & $0.11(1.14)$ & $0.06(0.54)$ & $-0.53(0.88)$ \\
\hline
\end{tabular}

were found at frontal electrode positions $\left(F_{1,23}=5.93 ; p<.05\right)$; however, the effect was only marginally significant at central electrode positions $\left(F_{1,23}=3.57 ; p=.07\right)$ and no difference of the P2 elicited by the familiar and the unfamiliar stimulus was found at posterior electrodes.

Thirdly, the familiar stimulus elicited a more positive ERP in a time window subsequent to the P2 maximum, which affected the terminating slope of the P2. This positive deflection was visible as a distinct peak at a latency around $230 \mathrm{~ms}$ in the familiarminus-unfamiliar difference wave (see Fig. 3, gray line). This indicated that the deflection was a distinct component rather than a modulation of the original P2. Following Garcia-Larrea et al. (1992), who found a similar positive deflection in the terminating slope of the P2, this deflection was labeled as P250 (cf. Garcia-Larrea et al., 1992). Although observed in a time window almost identical to that of the P3a observed in the Location-oddball condition, the P250 effect can be differentiated from that P3a by, firstly, being observed in the ERP to standard or non-deviant sounds instead of deviants, and, secondly, by showing a more frontally oriented scalp distribution (cf. Fig. 4). The ANOVA for the P250 time window

\section{ERPs}

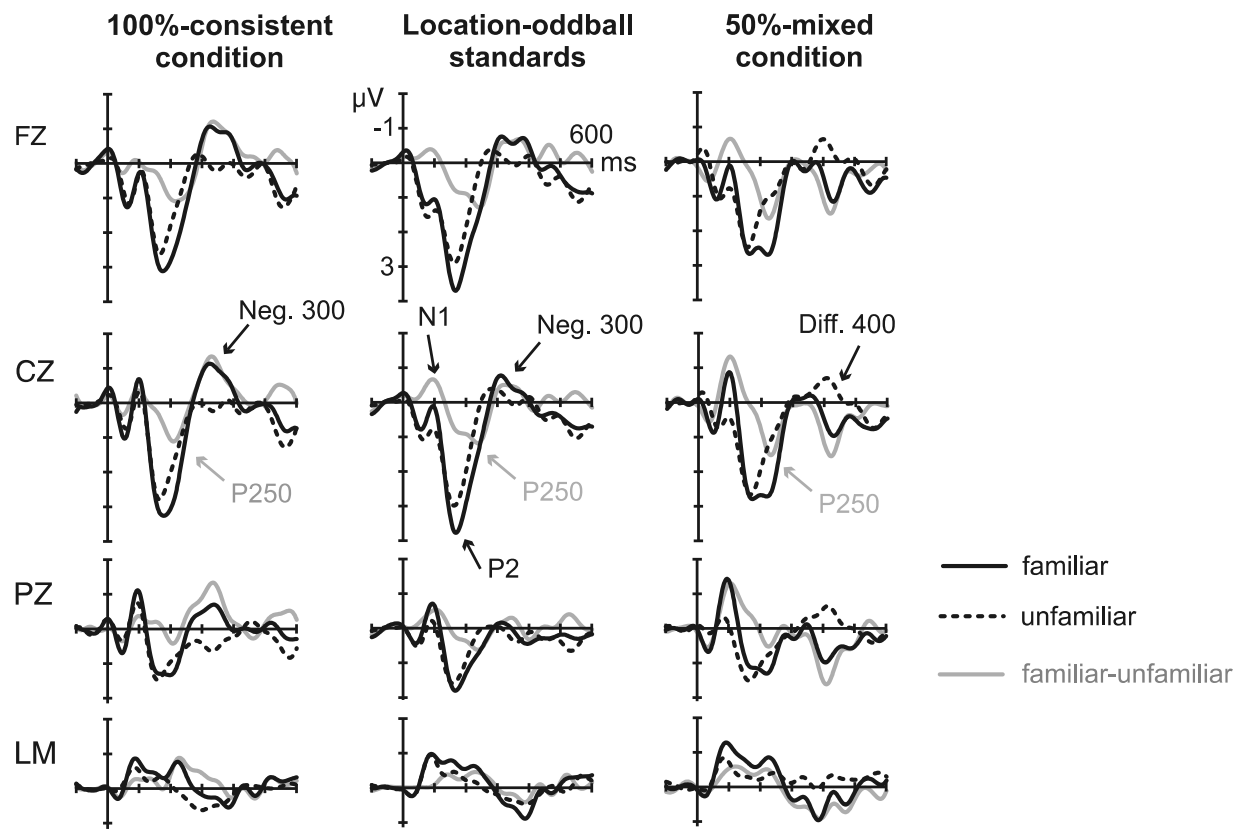

Fig. 3. ERPs elicited by the familiar and the unfamiliar stimulus in the three conditions (deviants from the Location-oddball condition were not included in the statistical analysis). 


\section{Scalp distributions of familiarity effects}

(familiar-minus-unfamiliar difference maps)

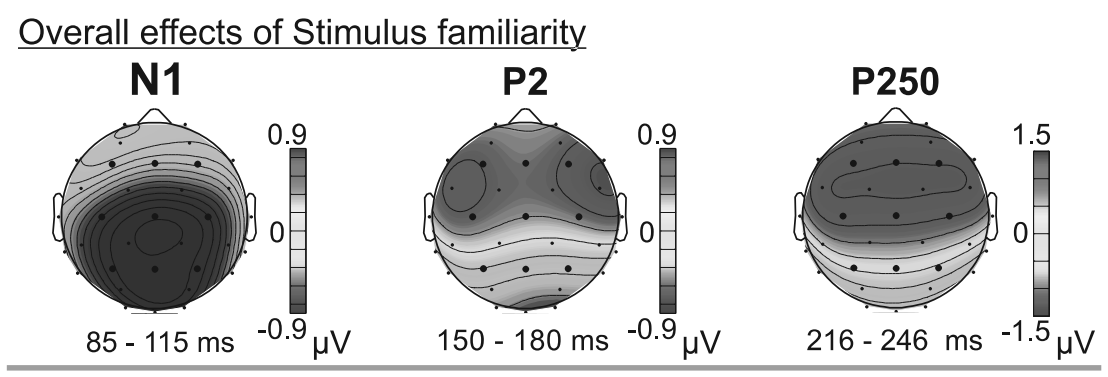

Stimulus familiarity $x$ Condition

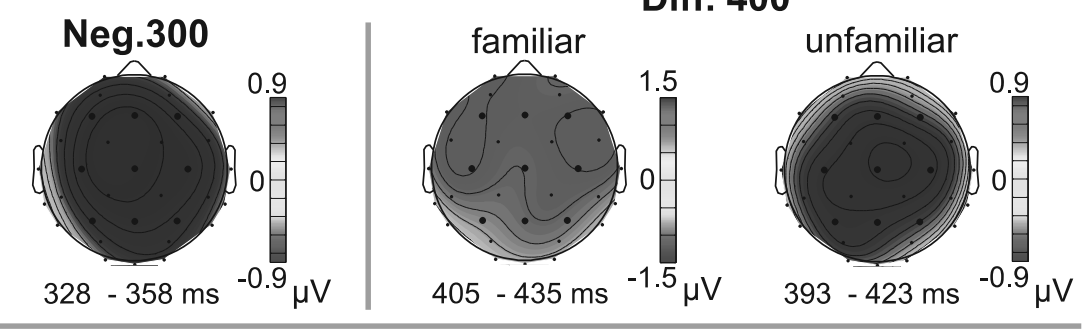

Fig. 4. Voltage distributions for the effects of sound familiarity observed in the familiar-minus-unfamiliar difference wave. Difference maps were averaged over the conditions contributing to the respective effect as indicated by the statistical analysis [N1/P2/P250 - average over all conditions; Neg.300 - average over 100\%-consistent condition and Location-oddball standards; Diff.400 - 50\%-mixed condition]. Maps for the Diff. 400 are displayed with separate time windows for the familiar and the unfamiliar stimulus to optimally display both subcomponents of the effect. However, statistical testing of the Diff.400 with separate time windows did not yield different results. Larger dots indicate the electrode position included the in statistical analysis.

yielded a main effect of Stimulus familiarity $\left(F_{1,23}=28.05\right.$; $p<.001)$. Moreover, an interaction of Stimulus familiarity $\mathrm{x}$ Anteriority $\left(F_{2,46}=23.33 ; p<.001\right)$ reflected a frontally oriented maximum of the effect (cf. Fig. 4). Reduced ANOVAs yielded significant effects of Stimulus familiarity at frontal and central electrode positions (frontal: $F_{1,23}=35.51 ; p<.001$; central: $F_{1,23}=34.47 ; p<.001$ ), but not over posterior regions.

The observed N1, P2 and P250 effects did not statistically interact with the factor Condition and were thus not sensitive to the sound context. Additionally, although temporal overlap with the deviance-related components does not allow statistical analysis of the respective time windows for the deviant stimuli, similar effects of sound familiarity were visible for the familiar in contrast to the unfamiliar deviant stimulus (cf. Fig. 5). Thus, the observed effects can be taken to reflect overall effects of sound familiarity in the present study. Taken together, the enhanced N1, P2 and P250 components reflect enhanced processing of the familiar stimulus before $300 \mathrm{~ms}$ after stimulus onset.

\subsection{Location deviant processing}

Deviant-related ERP effects are displayed in Fig. 5 (cf. Table 2). Deviants in sound location elicited a significant MMN at a peak latency of $130 \mathrm{~ms}$ (main effect Oddball stimulus; $F_{1,23}=88.74$; $p<.001$ ), as well as a significant P3a at a peak latency of $233 \mathrm{~ms}$ (main effect Oddball stimulus; $F_{1,23}=32.13 ; p<.001$; see Fig. 5a). For the MMN, an Oddball stimulus $\times$ Anteriority interaction $\left(F_{2.46}=17.91 ; p<.001\right)$ reflected a central maximum of the response (cf. Fig. $5 b$ ). For the P3a, interactions of Oddball stimulus $\times$ Laterality $\left(F_{2.22}=14.61 ; p<.001\right)$, Oddball stimulus $\times$ Anteriority $\left(F_{2,46}=12.14 ; p=.001\right)$ and Oddball stimulus $\times$ Laterality $\times$ Anteriority $\left(F_{4,20}=3.25 ; p<.05\right)$ reflected a maximum of the P3a over the vertex (cf. Fig. 5b). No interactions with the factor Familiarity were observed. Solely, a four-way interaction of Oddball stimulus $\times$ Familiarity $\times$ Laterality $\times$ Anteriority $\left(F_{4,20}=3.39\right.$; $p<.05)$ indicated a slightly broader extension of the P3a to right-central and left-posterior areas for the unfamiliar stimulus (cf. Fig. 5b). This, however, was due to a sustained, right-parietal negativity observed for the familiar stimulus in the deviantminus-standard difference wave between 250 and $500 \mathrm{~ms}$ (cf. Fig. 5). This negative deflection elicited for the familiar deviant was reflected in a significant Oddball stimulus $\times$ Familiarity $\times$ Anteriority interaction in the respective time window $\left(F_{2,46}=9.02 ; p<.01\right)$. The negativity showed an N400-like morphology (for a review on the N400 see Kutas and Federmeier, 2000) and was not observed in the unfamiliar deviant-minus-standard difference wave.

\subsection{ERP modulations depending on the sound context}

Additionally, familiarity-specific ERP modulations as an effect of the sound context were observed in two time windows subsequent to $300 \mathrm{~ms}$ (see Figs. 3 and 4, cf. Table 1 ).

Firstly, when a consistently familiar sound context was present, i.e. for the familiar stimulus in the $100 \%$-consistent condition and familiar Location-oddball standards, the familiar stimulus elicited a widely distributed, negative ERP deflection between 300 and 400 ms (labeled as Neg.300, cf. Figs. 3 and 4). The ANOVA in the respective time window yielded an interaction of Stimulus familiarity $\times$ Condition $\left(F_{2,46}=4.18 ; p<.05\right)$, that drove an additional main effect of Stimulus familiarity $\left(F_{1,23}=6.92 ; p<.05\right)$. On the one hand, the interaction reflected a significant effect of Condition for the familiar (familiar: $F_{2.22}=3.69 ; p<.05$ ), but not the unfamiliar stimulus. Post-hoc comparisons for the familiar stimulus yielded a more negative ERP in the $100 \%$-consistent compared to the $50 \%$-mixed condition $(p<.05)$, whereas the familiar Location-oddball standards did not differ from either condition. On the other hand, reduced ANOVAs yielded a significant effect of Stimulus familiarity in the $100 \%$-consistent condition $\left(F_{1,23}=6.89 ; p<.05\right)$ and for the Location-oddball standards 


\section{Deviance-related processing}

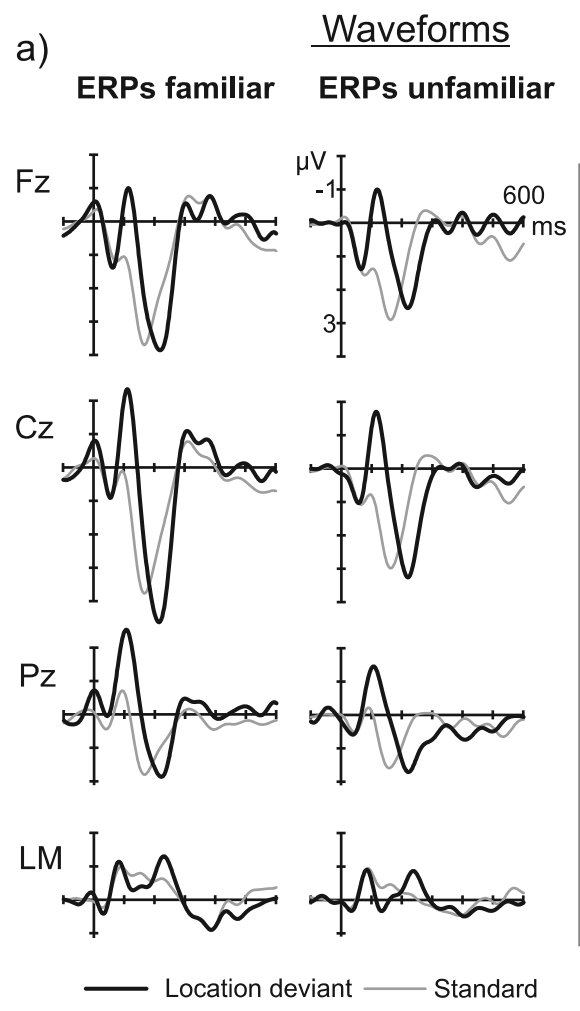

Deviant-minus-

b) Scalp distributions standard

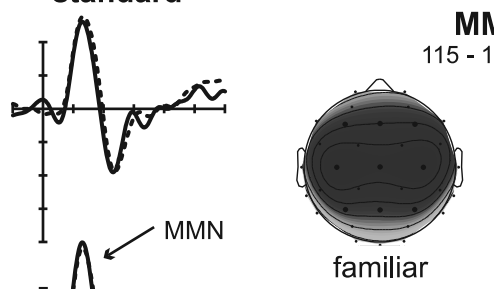

MMN

115 - $145 \mathrm{~ms}$

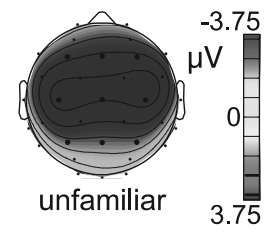

P3a

$218-248 \mathrm{~ms}$

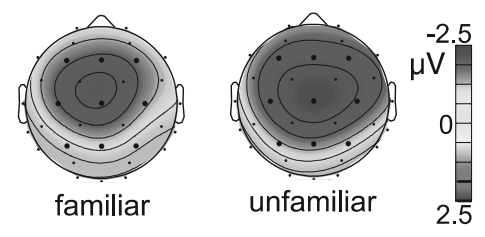

N400

$300-400 \mathrm{~ms}$

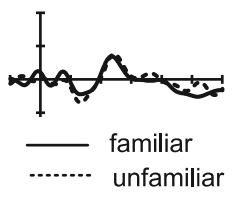

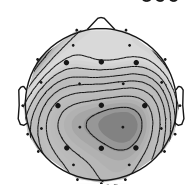

familiar

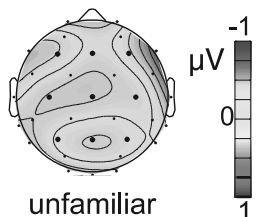

Fig. 5. Deviance-related effects. Voltage maps (b) show the respective distribution of the deviance-related effects separately for the familiar and the unfamiliar stimulus (deviant-minus-standard difference maps). Larger dots indicate the electrode position included in the statistical analysis.

$\left(F_{1,23}=9.68 ; p<.01\right)$, but not in the $50 \%$-mixed condition. Although not tested statistically, a similar negative deflection was observed when comparing familiar and unfamiliar deviant sounds (cf. Fig. 5a). Taken together, an additional negative deflection between 300 and 400 ms was observed for the familiar stimulus in a consistently familiar sound context.

Conversely, differential processing of the familiar and the unfamiliar stimuli was observed in a time range between 400 and $500 \mathrm{~ms}$ (labeled as Diff. 400, cf. Fig. 3) when both stimuli occurred intermixed (i.e. in the 50\%-mixed condition). The respective ANOVA yielded a main effect of Stimulus familiarity $\left(F_{1,23}=8.67 ; p<.01\right)$, which was driven by an interaction of Stimulus familiarity $\times$ Condition $\left(F_{2,46}=9.31 ; p=.001\right)$. Reduced ANOVAs showed a two-fold effect: For the familiar stimulus, an effect of Condition $\left(F_{2,46}=7.68 ; p<.01\right)$ reflected a widespread positive deflection elicited in the 50\%-mixed condition, but not in the conditions with a consistently familiar context (comparison to $100 \%$ consistent condition: $p<.05$; comparison to Location-oddball standards: $p<.01)$. Contrariwise, an effect of Condition for the unfamiliar stimulus $\left(F_{2,22}=6.33 ; p<.01\right)$ reflected a widespread negative deflection in the ERP for the unfamiliar stimulus in the 50\%-mixed condition, which was not observed when the context was consistently unfamiliar (comparison to $100 \%$-consistent condi-

Table 2

Mean amplitudes (standard deviations) of the deviance-related effects for the familiar and the unfamiliar stimulus.

\begin{tabular}{lllr}
\hline & MMN & P3a & \multicolumn{1}{l}{ N400 } \\
\hline familiar & $-2.68(1.69)$ & $1.30(1.95)$ & $-.17(1.28)$ \\
unfamiliar & $-2.48(1.46)$ & $1.61(1.20)$ & $.20(1.38)$ \\
\hline
\end{tabular}

tion: $p<.05$; comparison to Location-oddball standards: $p<.01$ ). Accordingly, a significant dissociation of familiar and unfamiliar stimulus processing between 400 and $500 \mathrm{~ms}$ was only observed in the $50 \%$-mixed condition $\left(F_{1,23}=29.48 ; p<.001\right)$. This effect thus exclusively occurred when both stimuli occurred together.

\section{Discussion}

The present study investigated three aspects of sound familiarity effects on environmental sound processing outside the focus of attention. The results will be discussed separately regarding: (1) overall processing differences between the familiar and the unfamiliar sound, (2) location-deviant processing for the familiar and the unfamiliar sound and (3) familiarity-specific processing depending on the sound context.

\subsection{Overall processing differences between the familiar and the unfamiliar sound}

In general, the familiar sound elicited additional stimulus processing before $300 \mathrm{~ms}$. This was manifest in a posteriorly enhanced N1 component, a frontally enhanced P2 and an additional P250 component following the P2 in the ERP elicited by the familiar stimulus. Thus, processing advantages for the familiar sound can be concluded already on relatively low-level processing stages and even when sounds were processed outside the focus of attention.

An enhancement of the $\mathrm{N} 1$ and $\mathrm{P} 2$ by sound familiarity is in line with previous results reporting enhanced $\mathrm{N} 1(\mathrm{~m})$ and/or P2 responses when listeners were familiar with speech sounds or musical instrument sounds (Pantev et al., 1998; see also Shahin et al., 
2003; Ylinen and Huotilainen, 2007; see also e.g., Tremblay and Kraus, 2002). For the first time, the present study, showed similar results of acoustic familiarity for a complex environmental sound associated with a semantic long-term memory representation.

Notably, the observed effects occur in a time window in which a central sound representation is established (cf. Näätänen, 1992; Näätänen and Winkler, 1999). Pantev et al. (1998) proposed that enhanced responses of the auditory $\mathrm{N} 1 \mathrm{~m}$ for familiar musical sounds, either reflecting increased recruitment of neural populations or better synchronization of their neural activity, might reflect enhanced cortical representations for familiar auditory events. The observed N1-P2 effects in the present study thus might reflect the establishment of a more elaborated sensory memory representation for the familiar environmental sound. However, the surface-recorded auditory N1 is no unitary phenomenon, but receives contributions from at least three distinct generator structures (cf. Näätänen and Picton, 1987). Whereas the supratemporal $\mathrm{N} 1$ generator is mainly sensitive to physical stimulus properties, which were thoroughly controlled in the present study, additional "unspecific" N1 generators were proposed to be sensitive to more elaborate stimulus features or even motivational states (cf. Näätänen and Picton, 1987) ${ }^{3}$. Although the earliness of the effects might indicate that first effects of sound familiarity were generated in auditory cortex, the posterior maximum of the $\mathrm{N} 1$ effect does not point to a source in the primary auditory cortex (i.e. the supratemporal N1 generator), which should cause frontocentrally maximal effects. This is in line with Altmann et al. (2007) who proposed a processing of animal vocalizations in putatively nonprimary auditory areas of the superior temporal gyrus (cf. also Lewis et al., 2004). Whereas Altmann et al. (2007) as well as Lewis et al. (2004) further reported a left-hemispheric predominance in the processing of animal sounds or familiar environmental sounds in fMRI data, no lateralization effects were observed in the present study. The posterior distribution of the $\mathrm{N} 1$ familiarity effect could reflect the activation of additional cortical generator structures for a familiar environmental sound (for example, the anterior-temporal cortex that has been proposed to be critical for the activation of semantic knowledge representations; cf. Damasio et al., 1996; Kotz et al., 2007; cf. also Murray et al., 2008). On the other hand, it could indicate an autonomous effect of sound familiarity overlaying the N1 in time. Roye et al. (2007) observed a posteriorly distributed negativity in response to personally significant in contrast to personally non-significant deviant ringtones, i.e. when using a different approach to manipulate sound familiarity. Whereas Roye et al. (2007) reported an effect around $200 \mathrm{~ms}$, the present study used a more distinct manipulation of sound familiarity, which might have contributed to earlier effects.

The observed P250 is similar to a component observed by Garcia-Larrea et al. (1992). The authors proposed this component to reflect a rejection of task-irrelevant, non-target stimuli from further processing. A familiar environmental sound might involuntarily be processed more elaborately and might require increased rejection from further processing to preserve processing resources. It cannot be excluded that the positivity was related to the P3a or other attention-related positivities in this time window; however its morphology was clearly dissociated from the P3a. Generally, the ERPs do not indicate differences in the allocation of attention to the familiar and the unfamiliar stimulus.

Summarizing the effects before $300 \mathrm{~ms}$, the present data speak for enhanced processing of a complex sound represented in auditory long-term memory. This enhanced processing was observed already on low-level processing stages and even though sounds

\footnotetext{
${ }^{3}$ When using a physically very similar stimulus manipulation as applied in the present study, Lenz et al., 2007 did not observe differences in the N1. In their study, sounds were processed attentively, which indicates crucial differences between sound familiarity effects in passive vs. attentive processing.
}

were processed outside the focus of attention. Such enhanced processing could constitute the foundation for a rapid semantic analysis of meaningful environmental sounds when sounds get behaviorally relevant. Data from the visual modality propose that familiar items rapidly activate associations in a specific Conceptual Short-term Memory (CSTM, cf. Potter, 1993; see also e.g., O'Connor and Potter, 2002). This might similarly apply for the auditory modality. According to the present data, more processing resources seem to be involuntarily recruited for the processing of a familiar environmental sound.

\subsection{Location-deviant processing for the familiar and the unfamiliar sound}

For the processing of deviants in sound location, very similar deviance-related processing was observed for the familiar and the unfamiliar stimulus. As expected, location deviants elicited an MMN, indicating the detection of the location change (e.g., Schröger, 2007), and a P3a, indicating an involuntary switch of attention to the location change (e.g., Escera, 1998). However, whereas several previous studies observed familiarity effects on the MMN and/or P3a (e.g., Näätänen et al., 1997; Beauchemin et al., 2006; Pulvermüller et al., 2001; Jacobsen et al., 2004, 2005), no effects of sound familiarity on these deviant-related components were observed in the present study. Note, that an effective manipulation of sound familiarity was assured in a stimulus pre-test and was reflected in overall processing differences observed for the familiar and the unfamiliar stimulus. Moreover, in the present design, not only deviant but also standard stimuli differed in their familiarity, which should have maximized familiarity-related effects on auditory deviance processing (cf. Jacobsen et al., 2004, 2005).

The design of the present study differed crucially from previous studies that addressed sound familiarity effects on auditory deviance processing. In the present study, the deviant was the same sound as the corresponding standard and the deviance was a change in a very basic auditory feature, which was entirely unrelated to the familiarity of the sounds. Previous studies used deviants and standards that were different auditory objects (e.g., Beauchemin et al., 2006; Frangos et al., 2005) - in this case, the familiarity of the respective sounds might directly influence the sensory memory comparison reflected in the MMN. The familiarity effects observed before $300 \mathrm{~ms}$ in the present study indicate that familiar sounds are already processed differently on low-level auditory processing stages, which could result in an increased dicriminability of familiar sounds. This would enhance deviancerelated components (e.g., Atienza et al., 2002). It seems that no familiarity-related effects on auditory deviance processing are triggered when the deviance is unrelated to the familiarity of the sounds. Rather, a deviance might need to include a change of the auditory item itself in order to trigger such effects. The absence of familiarity effects in the present study might also be locationspecific, as several data proposed distinct processing pathways of auditory "what" and "where" information similarly as in the visual modality (e.g., Rauschecker, 1998; Alain et al., 2001).

Subsequently to the deviance-related components, deviants in sound location notably triggered additional semantic analysis of the familiar stimulus, as indicated by an N400 elicited by familiar deviants but not standards (for N400 elicited by environmental sounds see e.g., van Petten and Rheinfelder, 1995; Cummings et al., 2006; Orgs et al., 2007; for a review on the N400 see Kutas and Federmeier, 2000). Typically, the N400 is not elicited when sounds are processed outside the focus of attention (cf. Mecklinger et al., 1997; Escera et al., 2003). Correspondingly, the present data only indicate a semantic analysis of the familiar stimulus after a physical deviance attracted attention to the sounds. It thus can 
be assumed that familiarity effects observed for the unattended standards do not reflect the semantic content, but rather the acoustic familiarity of the familiar sound.

\subsection{Familiarity-specific processing depending on the sound context}

Two ERP modulations reflected familiarity-specific processing depending on the sound context after $300 \mathrm{~ms}$. In this time range, ERP effects usually indicate higher-order cognitive processes rather than processing related to specific physical features of a sensory stimulus. As the identical stimuli were presented in all three conditions, processing differences for the familiar and the unfamiliar sound over the conditions must reflect an influence of the overall sound context.

When the familiar sound was presented in a context of familiar sounds only, it elicited an additional negative deflection between 300 and $400 \mathrm{~ms}$ compared to when presented intermixed with the unfamiliar environmental sound (Neg.300). In contrast, presenting the unfamiliar stimulus in a context of consistently unfamiliar stimuli did not yield a homologous effect. The Neg.300 effect thus cannot be merely explained by a repetitive stimulus presentation, but occurs specifically for the familiar sound. The Neg.300 might be related to the N2c, a component that has been proposed to reflect processes related to stimulus classification (cf. Pritchard et al., 1991). In the present study, the Neg.300 indicated sustained stimulus processing for a familiar sound in a consistently familiar sound sequence.

Conversely, an additional processing dissociation between the familiar and the unfamiliar stimulus was observed between 400 and $500 \mathrm{~ms}$ when both stimuli were presented together in one block. In this time window, the ERP for the familiar stimulus showed an additional positive deflection, while the ERP for the unfamiliar stimulus showed an additional negative deflection. No difference was observed in this time window when both sounds were presented in separate blocks. The observed bi-directional dissociation thus indicates additional stimulus classification processes when two distinct sounds are presented in a common sequence. However, as only two stimuli were presented in the present study, these classification processes might have equally related to the difference in sound familiarity or the general dissimilarity of both sounds (for related effects in this time window see Muller-Gass et al., 2007). Even though the observed effect might not necessarily be familiarity-specific, interestingly, in this time window, different processes yet seem to be triggered for the familiar and the unfamiliar stimulus.

The observed context-sensitive effects occur in a time window associated with semantic integration processes (N400; cf. Kutas and Federmeier, 2000) and with retrieval processes from auditory long-term memory (gamma-band response, cf. Lenz et al., 2007). Semantic integration processes are unlikely to explain the observed effects, given the highly repetitive sound sequences, stimulus processing outside the focus of attention (cf. Mecklinger et al., 1997) and lacking similarity with the N400 (cf. Kutas and Federmeier, 2000). Moreover, semantic analysis of the presented sounds was only indicated after an involuntary switch of attention to the sounds. On the other hand, long-term memory retrieval processes (cf. Lenz et al., 2007) might enable stimulus-context matching processes. Taken together, ERP effects observed after $300 \mathrm{~ms}$ reflected familiarity-specific sound processing depending on the sound context.

\subsection{Conclusion}

The present study showed effects of sound familiarity on the processing of a complex environmental sound outside the focus of attention. Generally, effects were observed in two distinct time ranges. On the one hand, preferential processing due to acoustic long-term familiarity was elicited for the familiar environmental sound before $300 \mathrm{~ms}$. A foundation for rapid extraction of meaning from a familiar environmental sound thus is indicated to be established relatively early and even when sounds are not consciously attended. On the other hand, the absence of familiarity-related effects on the processing of deviants in sound location suggests that a deviance has to relate to the familiarity of the sounds in order to yield familiarity-related effects on auditory deviance processing. After $300 \mathrm{~ms}$, ERPs reflected familiarity-specific processing depending on the sound context.

\section{Acknowledgement}

The authors thank Prof. István Winkler for comments on a previous version of this manuscript and Andreas Widmann as well as the auditory list (http://www.auditory.org) for support in the development of the stimulus manipulation algorithm. Ursula Kirmse was supported by the German Research Council (DFG), as part of the Research Training Group 1182, "Function of attention in cognition”. Erich Schröger is supported by a DFG-Reinhart KoselleckProject.

\section{References}

Alain C, Arnott SR, Hevenor S, Graham S, Grady CL. What and where in the human auditory system. Proc Natl Acad Sci USA 2001;98(21):12301-6.

Altmann CF, Doehrmann O, Kaiser J. Selectivity for animal vocalizations in the human auditory cortex. Cereb Cortex 2007;17(11):2601-8.

Atienza M, Cantero JL, Dominguez-Marin E. The time course of neural changes underlying auditory perceptual learning. Learning Mem 2002;9(3):138-50.

Ballas JA, Howard JH. Interpreting the language of environmental sounds. Environ Behav 1987:19(1):91-114.

Ballas JA, Mullins T. Effects of context on the identification of everyday sounds. Hum Perform 1991;4(3):199-219.

Beauchemin M, De Beaumont L, Vannasing P, Turcotte A, Arcand C, Belin P, et al. Electrophysiological markers of voice familiarity. Eur J Neurosci 2006:23(11):3081-6

Bosnyak DJ, Eaton RA, Roberts LE. Distributed auditory cortical representations are modified when non-musicians are trained at pitch discrimination with $40 \mathrm{~Hz}$ amplitude modulated tones. Cereb Cortex 2004;14(10):1088-99.

Cummings A, Ceponiene R, Koyama A, Saygin AP, Townsend J, Dick F. Auditory semantic networks for words and natural sounds. Brain Res 2006:1115 92-107.

Cycowicz YM, Friedman D. Effect of sound familiarity on the event-related potentials elicited by novel environmental sounds. Brain Cogn 1998;36(1):30-51.

Damasio H, Grabowski TJ, Tranel D, Hichwa RD, Damasio AR. A neural basis for lexical retrieval. Nature 1996;380(6574):499-505.

Escera C, Alho K, Winkler I, Näätänen R. Neural mechanisms of involuntary attention to acoustic novelty and change. J Cogn Neurosci 1998;10(5):590-604.

Escera C, Yago E, Corral MJ, Corbera S, Nunez MI. Attention capture by auditory significant stimuli: semantic analysis follows attention switching. Eur J Neurosci 2003;18(8):2408-12.

Frangos J, Ritter W, Friedman D. Brain potentials to sexually suggestive whistles show meaning modulates the mismatch negativity. Neuroreport 2005;16(12):1313-7.

Garcia-Larrea L, Lukaszewicz AC, Mauguiere F. Revisiting the oddball paradigm nontarget vs neutral stimuli and the evaluation of Erp attentional effects. Neuropsychologia 1992;30(8):723-41.

Holeckova I, Fischer C, Giard MH, Delpuech C, Morlet D. Brain responses to a subject's own name uttered by a familiar voice. Brain Res 2006:1082 142-152.

Horvath J, Winkler I, Bendixen A. Do N1/MMN, P3a, and RON form a strongly coupled chain reflecting the three stages of auditory distraction? Biol Psychol 2008;79(2):139-47.

Jacobsen T, Horvath J, Schröger E, Lattner S, Widmann A, Winkler I. Pre-attentive auditory processing of lexicality. Brain Lang 2004;88(1):54-67.

Jacobsen T, Schröger E, Winkler I, Horvath J. Familiarity affects the processing of task-irrelevant auditory deviance. J Cogn Neurosci 2005;17(11):1704-13.

Kotz SA, Opitz B, Friederici AD. ERP effects of meaningful and non-meaningful sound processing in anterior temporal patients. Restor Neurol Neurosci 2007;25(3-4):273-84

Kuriki S, Kanda S, Hirata Y. Effects of musical experience on different components of MEG responses elicited by sequential piano-tones and chords. J Neurosci 2006;26(15):4046-53.

Kutas M, Federmeier KD. Electrophysiology reveals semantic memory use in language comprehension. Trends Cogn Sci 2000;4(12):463-70. 
Lenz D, Schadow J, Thaerig S, Busch NA, Herrmann CS. What's that sound? Matches with auditory long-term memory induce gamma activity in human EEG. Int J Psychophysiol 2007;64(1):31-8.

Lewis JW, Wightman FL, Brefczynski JA, Phinney RE, Binder JR, DeYoe EA. Human brain regions involved in recognizing environmental sounds. Cereb Cortex 2004;14(9):1008-21.

Lewis JW, Brefczynski JA, Phinney RE, Janik JJ, DeYoe EA. Distinct cortical pathways for processing tool versus animal sounds. J Neurosci 2005;25(21):5148-58.

Mecklinger A, Opitz B, Friederici AD. Semantic aspects of novelty detection in humans. Neurosci Lett 1997;235(1-2):65-8.

Menning $\mathrm{H}$, Roberts LE, Pantev C. Plastic changes in the auditory cortex induced by intensive frequency discrimination training. Neuroreport 2000;11(4):817-22.

Muller-Gass A, Roye A, Kirmse U, Saupe K, Jacobsen T, Schroger E. Automatic detection of lexical change: an auditory event-related potential study. Neuroreport 2007;18(16):1747-51.

Murray MM, Camen C, Spierer L, Clarke S. Plasticity in representations of environmental sounds revealed by electrical neuroimaging. Neuroimage 2008;39(2):847-56

Näätänen R. Attention and brain function. Hillsdale: Erlbaum; 1992.

Näätänen R, Lehtokoski A, Lennes $M$, Cheour $M$, Huotilainen $M$, livonen $A$, et al. Language-specific phoneme representations revealed by electric and magnetic brain responses. Nature 1997;385(6615):432-4

Näätänen R, Paavilainen P, Rinne T, Alho K. The mismatch negativity (MMN) in basic research of central auditory processing: A review. Clin Neurophysio 2007;118(12):2544-90.

Näätänen R, Picton T. The N1 wave of the human electric and magnetic response to sound - a review and an analysis of the component structure. Psychophysiology 1987;24(4):375-425.

Näätänen $R$, Winkler I. The concept of auditory stimulus representation in cognitive neuroscience. Psychol Bull 1999;125(6):826-59.

Neuloh G, Curio G. Does familiarity facilitate the cortical processing of music sounds? Neuroreport 2004;15(16):2471-5.

O'Connor KJ, Potter MC. Constrained formation of object representations. Psycho Sci 2002;13(2):106-11.

Orgs G, Lange K, Dombrowski JH, Heil M. Is conceptual priming for environmental sounds obligatory? Int J Psychophysiol 2007;65(2):162-6.

Pantev C, Oostenveld R, Engelien A, Ross B, Roberts LE, Hoke M. Increased auditory cortical representation in musicians. Nature 1998;392(6678):811-4.

Pantev C, Roberts LE, Schulz M, Engelien A, Ross B. Timbre-specific enhancement of auditory cortical representations in musicians. Neuroreport 2001;12(1): 169-74.
Potter MC. Very short-term conceptual memory. Mem Cognit 1993;21(2):156-61. Pritchard WS, Shappell SA, Brandt ME. Psychophysiology of N200/ N400: a review and classification scheme. In: Jennings JR, Ackles PK, Coles MGH, editors. Advances in psychophysiology: a research annual. London: Jessica Kingsley; 1991. p. 43-106.

Pulvermüller F, Kujala T, Shtyrov Y, Simola J, Tiitinen H, Alku P, et al. Memory traces for words as revealed by the mismatch negativity. Neuroimage $2001 ; 14(3)$ : 607-16.

Rauschecker JP. Parallel processing in the auditory cortex of primates. Audiol Neurootol 1998;3(2-3):86-103.

Roye A, Jacobsen T, Schröger E. Personal significance is encoded automatically by the human brain: an event-related potential study with ringtones. Eur J Neurosci 2007;26(3):784-90.

Schröger E. Mismatch negativity - a microphone into auditory memory. J Psychophysiol 2007;21(3-4):138-46.

Shahin A, Bosnyak DJ, Trainor LJ, Roberts LE. Enhancement of neuroplastic P2 and N1c auditory evoked potentials in musicians. J Neurosci 2003;23(13): 5545-52.

Shahin A, Roberts LE, Trainor LJ. Enhancement of auditory cortical development by musical experience in children. Neuroreport 2004;15(12):1917-21.

Sheehan KA, McArthur GM, Bishop DVM. Is discrimination training necessary to cause changes in the P2 auditory event-related brain potential to speech sounds? Cogn Brain Res 2005;25(2):547-53.

Sussman E, Winkler I. Dynamic sensory updating in the auditory system. Cogn Brain Res 2001;12(3):431-9.

Trainor LJ, Trehub SE. Musical context effects in infants and adults: key distance. J Exp Psychol Hum Percept Perform 1993;19(3):615-26.

Tremblay KL, Kraus N. Auditory training induces asymmetrical changes in cortica neural activity. J Speech Lang Hear Res 2002;45(3):564-72.

van Petten C, Rheinfelder $\mathrm{H}$. Conceptual relationships between spoken words and environmental sounds - event-related brain potential measures. Neuropsychologia 1995;33(4):485-508.

Wetzel N, Schröger E. Modulation of involuntary attention by the duration of nove and pitch deviant sounds in children and adolescents. Biol Psychol 2007;75(1):24-31.

Winkler I. Interpreting the mismatch negativity. J Psychophysiol 2007;21(34):147-63.

Winkler I, Sussman E, Tervaniemi M, Horvath J, Ritter W, Näätänen R. Preattentive auditory context effects. Cogn Affect Behav Neurosci 2003;3(1):57-77.

Ylinen S, Huotilainen $\mathrm{M}$. Is there a direct neural correlate for memory-trace formation in audition? Neuroreport 2007;18(12):1281-4. 